Nota científica

(Short communication)

\title{
PRIMER REGISTRO PARA MÉXICO DE ALEUROPARADOXUS ARCTOSTAPHYLI RUSSELL, 1947 (HEMIPTERA: ALEYRODIDAE) SUS HOSPEDEROS, DISTRIBUCIÓN Y PARASITOIDE
}

\author{
FIRST RECORD FOR MÉXICO OF THE WHITEFLY ALEUROPARADOXUS \\ ARCTOSTAPHYLI RUSSELL, 1947, (HEMIPTERA: ALEYRODIDAE) \\ HOSTS, DISTRIBUTION AND PARASITOID
}

\author{
ÓSCAR ÁNgEL SÁNCHEZ-FLORES, ,** OSWALdo GARCÍA MARTÍNEZ1 \\ Y VICENTE EMILIO CARAPIA-RUIZ ${ }^{2}$
}

\footnotetext{
${ }^{1}$ Departamento de Parasitología Agrícola, Universidad Autónoma Agraria Antonio Narro, Calzada Antonio Narro No 1923, Colonia Buenavista, 25315, Saltillo, Coahuila, México Fax: (844) 4110228.<oscaruaaan@gmail.com>, $<$ drogarcía@yahoo.com.mx>

${ }^{2}$ Universidad Autónoma del Estado de Morelos (UAEM). Escuela de Estudios Profesionales de Xalostoc (EESuX). Av. Nicolas Bravo s/n, Parque Industrial Cuautla, Xalostoc, Ayala, Morelos. <vcarapia@hotmail.com> * autor para correspondencia: <oscaruaaan@gmail.com>

Recibido: 02/09/2016; aceptado: 16/01/2017

Editor responsable: Arturo Bonet
}

Sánchez-Flores, Ó. Á., García-Martínez, O. y Carapia-Ruiz, V. E. (2017) Primer registro para México de Aleuroparadoxus arctostaphyli Russell, 1947 (Hemiptera: Aleyrodidae), sus hospederos, distribución y parasitoide. Acta Zoológica Mexicana (n.s.), 33(1), 143-145.

RESUMEN. Se presenta el primer registro de Aleuroparadoxus arctostaphyli Russell en México con datos sobre sus hospederos, su distribución y un parasitoide.

En el mundo se han reportado diez especies del género Aleuroparadoxus (Martin y Mound, 2007) y para México, Russell (1947) reportó a A. gardeniae Russell, 1947 y Evans (2007) a A. gardeniae, A. ilicicola Russell, 1947, A. iridescens (Bemis), 1904, A. rhodae Russell 1947 y a A. truncatus Russell, 1947.

El 29 de enero de 2016, en el jardín botánico del Campus Buenavista-Saltillo de la Universidad Autónoma Agraria Antonio Narro (UAAAN), se recolectaron pupas de una especie de mosca blanca en el haz de hojas de Lindleya mespiloides, arbusto conocido como palo de pajarito, identificado en el herbario Antonio Narro Saltillo México (ANSM), las cuales se colocaron en cajas de Petri que fueron trasladadas, el mismo día, al Laboratorio
Sánchez-Flores, Ó. Á., García-Martínez, O., \& Carapia-Ruiz, V. E. (2017) First record for Mexico of the whitefly Aleuroparadoxus arctostaphyli Russell, 1947, (Hemiptera: Aleyrodidae), hosts, distribution and parasitoid. Acta Zoológica Mexicana (n.s.), 33(1), 143-145.

ABSTRACT. The first record for Mexico of the whitefly Aleuroparadoxus arctostaphyli Russell, with data on hosts, its distribution and a parasitoid is presented.

de Taxonomía de Insectos y Ácaros (LTIA) del Departamento de Parasitología Agrícola (DPA) de la UAAANSaltillo, para esperar la emergencia de parasitoides. Esta especie de mosca blanca ya había sido recolectada por Carapia-Ruíz. V. E. en Baja California Norte en hojas de un arbusto de la familia Ericaceae el 18 de diciembre de 2002, pero no la reportó. Solo emergió un parasitoide de las pupas, mismo que se montó en porta y cubreobjetos en bálsamo de Canadá, utilizando la técnica de Noyes (1982). Para la identificación del parasitoide a nivel de género, se utilizó la clave de Myartseva et al. (2009). Las pupas de la mosca blanca se procesaron mediante la técnica de Martin (2004) con algunas modificaciones; para determinar la especie de la mosca blanca, se utilizaron las 
claves de Russell (1947) y de Dooley (2010). Los ejemplares estudiados están depositados en el LTIA de la UAAAN-Saltillo.

La mosca blanca fue identificada como Aleuroparadoxus arctostaphyli Russell, 1947 y el parasitoide resultó ser una especie del genero Encarsia (Hymenoptera: Aphelinidae).

Diagnosis. Pupas sin procesar ni montar (Fig. 1a): color marrón oscuro a negro, elípticas a subcirculares, con pelos vidriosos cerosos que se extienden desde las papilas submarginales y dorsales. Pupas procesadas y montadas en portaobjetos (Fig. 1b): margen lateral. Área del poro traqueal indicada por 2-4 dientes marginales. Vista dorsal. Papilas submarginales de $32-56 \mu \mathrm{m}$, por lo general de 40 a $50 \mu \mathrm{m}$ de largo y 16-18 $\mu \mathrm{m}$ de ancho, no contiguas, la mayoría separadas unas de otras por una distancia de aproximadamente 1/5 de la anchura de una papila; el margen traqueal se pliega y termina dentado; fila de pa- pilas submarginales contigua. De 2 a 3 pares de papilas subdorsales modificadas en la parte cefálica; de cero a un par subdorsal central de papilas sobre el protórax; cada papila varía de 20 a $64 \mu \mathrm{m}$; cuatro pares de papilas reniformes dispersas presentes en el escudo cefálico. Orificio vasiforme (Fig. 1c). Es más bien ancho posteriormente, de 68-88 $\mu \mathrm{m}$ de largo y $60-80 \mu \mathrm{m}$ de ancho; opérculo de 64-80 $\mu \mathrm{m}$ de largo y 56-72 $\mu \mathrm{m}$ de ancho. Vista ventral. Antenas cortas con el ápice en forma de dedo que terminan bruscamente en T1; espinas obtusas presentes a lo largo del margen anterior de las coxas justo a la mitad de la banda rugosa; cada una de las patas medias y posteriores con una seta de cerca de $20 \mu \mathrm{m}$ de largo situada en la banda rugosa; poro traqueal torácico ausente. Quetotaxia. Cada seta cefálica y setas A1 y A8 abdominales de 24 a $32 \mu \mathrm{m}$ de longitud; setas caudales de 10 a $30 \mu \mathrm{m}$; setas abdominales ventrales de 35 a $40 \mu \mathrm{m}$ de longitud. (Dooley et al., 2010).

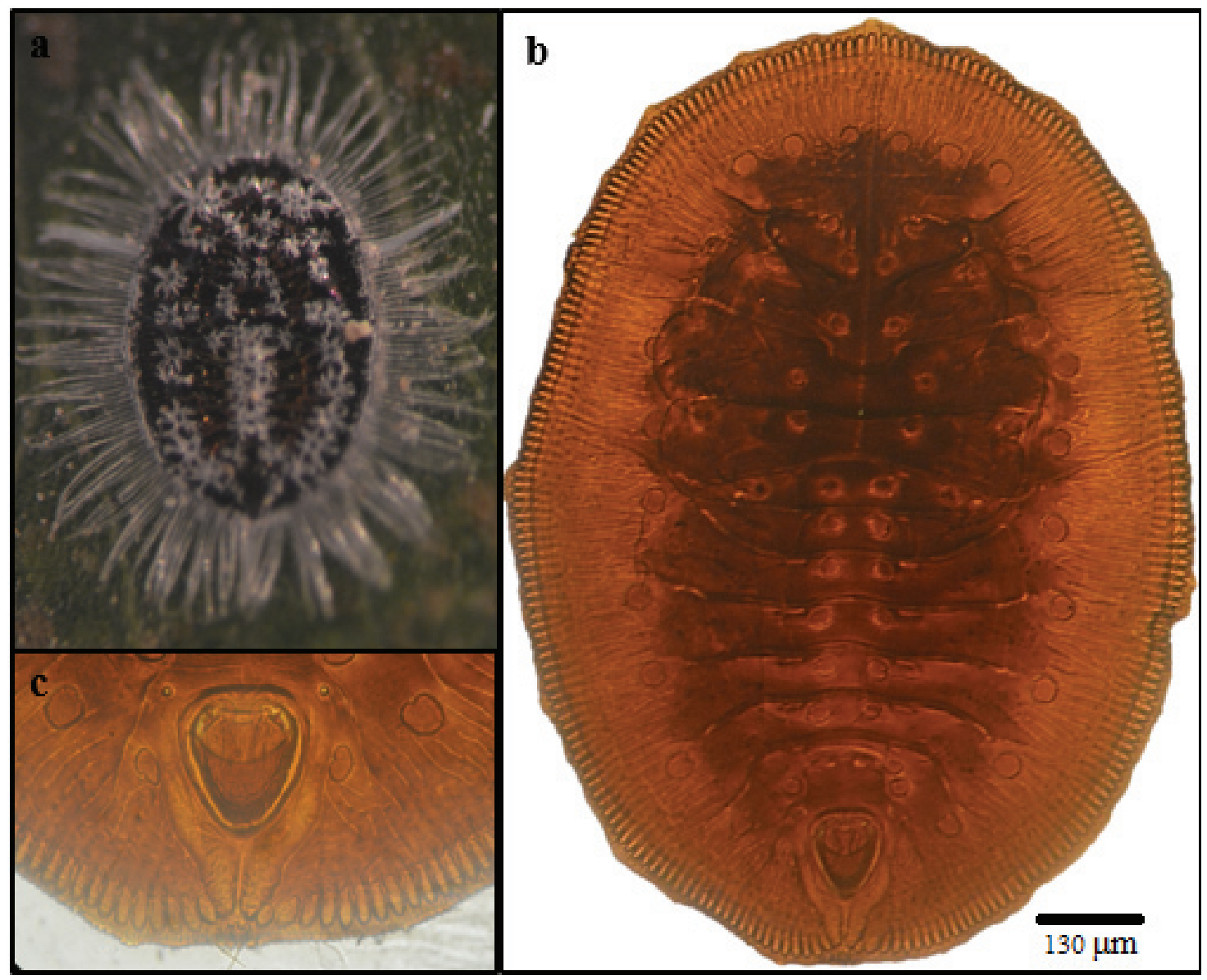

Figura 1. a) Pupa de Aleuroparadoxus arctostaphyli Russell, 1947 sin procesar ni montar, b) Pupas procesadas y montadas en portaobjetos, c) Orificio baciforme. 
Hospederos. Ericaceae: Arbutus menziesii, Arctostaphylos sp., A. manzanita, A. tomentosa, A. virgata, A. viscida; Rhamnaceae: Ceanothus sp. (Evans, 2007); Rosaceae: Lindleya mespiloides* Nuevo registro.

Distribución. Neártica USA (CA, OR, NV), (Evans, 2007); México (Baja California, Coahuila)* Nuevo registro.

Parasitoides. Hymenoptera: Aphelinidae; Encarsia sp.* Nuevo registro.

AGRADECIMIENTOS. A la Dra. Svetlana Nikolaevna Myartseva por confirmar que el parasitoide es una especie del género Encarsia. Al Dr. José Ángel Villareal Quintanilla por confirmar el hospedero.

\section{LITERATURA CITADA}

Dooley, J. W. III, Lambrecht, S. \& Honda, J. 2010. Eight new state records of Aleyrodine whiteflies found in Clark County, Nevada and three newly described taxa (Hemiptera: Aleyrodidae, Aleyrodinae). Insecta Mundi, Paper 660. http://digitalcommons.unl.edu/ insectamundi/660

Evans, G. A. 2007. The Whiteflies (Hemiptera: Aleyrodidae) of the world and their host plants and natural enemies. USDA/Animal Plant Health Inspection Service (APHIS) http://keys.lucidcentral. org/keys/v3/whitefly/PDF_PwP\%20ETC/world-whitefly-catalogEvans.pdf (acceso en agosto 2016).

Martin, J. H. 2004. Whiteflies of Belize (Homotera: Aleyrodidae) Part 1-Introduction and account of the subfamily Aleurodicinae Quaintance \& Baker. Zootaxa, 681, 1-119.

Martin, J. \& Mound, L. 2007. An annotated check list of the world's whiteflies (Insecta: Hemiptera: Aleyrodidae). Zootaxa 1492, 1-84.

Myartseva, S. N., Ruíz-Cancino, E. \& Coronado-Blanco, J. M. 2009. Identificación de los géneros de Aphelinidae (hymenoptera: Chalcidoidae), pp 935-939. In: Estrada-Venegas (Ed.). Entomología Mexicana. Vol. 8.

Noyes, J. S. 1982. Collecting and preserving chalcid wasps (Hymenoptera: Chalcidoidea). Journal of Natural History, 16, 215-33.

Russell, L. M. 1947. A classification of the whiteflies of the new tribe Trialeurodini (Homoptera: Aleyrodidae). Revista de Entomologia, Rio de Janeiro, 18, 1-44. 